\title{
Identification of Rare Polytypes of Presolar SiC with Coordinated TEM, Raman Spectroscopy and NanoSIMS Measurements
}

\author{
R. M. Stroud ${ }^{1}$, N. Liu ${ }^{2}$, Bradley De Gregorio ${ }^{1}$, A. Steele ${ }^{2}$, C. M O’D. Alexander ${ }^{2}$ and L. R. Nittler ${ }^{2}$ \\ 1. U.S. Naval Research Laboratory, Washington, DC 20375 \\ 2. Carnegie Institution of Washington, Washington, DC 20015
}

Silicon carbide is the most well-studied of presolar stardust phases. Transmission electron microscopy data from over 500 hundred presolar SiC grains, and secondary ion mass spectroscopy data from tens of thousands of grains, have been reported previously [1]. Despite the over 250 known synthetic polytypes, the only common polytypes of presolar SiC are $3 \mathrm{C}$ and $2 \mathrm{H}$, along with their intergrowths, some heavily disordered grains [2]. Evidence for the existence additional rare polytypes of presolar SiC based on analysis of the Raman TO position has recently been reported [3]. We seek to confirm the identification of these rare polytypes with electron diffraction, and ultimately look for correlations with stellar source, and/or subgrain content that could indicate different condensation conditions.

The grains for these studies were isolated from the Murchison meteorite using the CsF dissolution method, and dispersed onto gold foil. We then used the Carnegie Institution WiTec confocal Raman microscope with a $532 \mathrm{~nm}$ frequency-doubled Nd:YAG laser, to acquire spectra from 156 presolar SiC grains. In order to accurately measure small shifts in the Raman peak positions, we calibrated the spectrometer with external Neon and Ar-Hg lamps, and used synthetic $6 \mathrm{H} \mathrm{SiC}$ and BN grains as standards. NanoSIMS measurements of the C, Si and N isotope compositions were used to identify the stellar origin class of each grain. Sections of three grains were prepared for TEM analysis using focused ion beam microscopy with the Zeiss Auriga at CIW, and the FEI Nova 600 at NRL. TEM studies were performed using the NRL JEOL 2200FS TEM for selected area diffraction, and the Nion UltraSTEM 200-X with a 0.7sr Bruker SDD-EDS system for STEM imaging and elemental analysis, respectively. In order to minimize any variation in the camera constant during the diffraction imaging, care was taken to return the sample to the reference focus height after tilting, and fully spread the illumination before capturing diffraction patterns. The diffraction patterns were analyzed with the Calidris Crisp software to determine the peak positions, and angles. The Calidris PhIDO package was used to find possible zone matches for $3 \mathrm{C}, 2 \mathrm{H}, 4 \mathrm{H}, 6 \mathrm{H}, 8 \mathrm{H}, 15 \mathrm{R}, 21 \mathrm{R}$ and 33R polytypes, allowing for 3\% variability in d-spacings from the reference values, and $2^{\circ}$ angular variations. Crystal Maker Single Crystal Software was used to simulate diffraction patterns for comparison.

Our Raman data (Fig. 1) demonstrate that 84\% of the measured grains are consistent with the expected spectra for the predominant 3C polytype, whereas 9\% showed more complicated spectra either due to non-cubic polytypes or intergrowth, and $7 \%$ of grains had too weak a signal to make a polytype determination. Selected area diffraction of one grain (Fig. 2) with a distinctly non-cubic TO peak position confirms that it is inconsistent with the cubic polytype. Indexing of four distinct zone axis patterns reveals a best fit to the $8 \mathrm{H}$ polytype. Preliminary diffraction analysis of additional grains indicate the possibility of $6 \mathrm{H}$ and other higher order polytypes. Based on these analyses we conclude that coordinated Raman spectroscopy, TEM and NanoSIMS studies are an effective way to identify rare polytypes of $\mathrm{SiC}$ stardust in order to constrain models of stellar evolution. 
References:

[1] Nittler L. R. and Ciesla F. (2016) Annual Reviews in Astron. and Astrophys. 54, 53-93.

[2] Daulton T. L. et al. (2003) Geochem. Et Cosmochem. Acta 67, 4743-4767.

[3] Liu N. et al. (2017) Meteoritics and Planetary Science, in review.

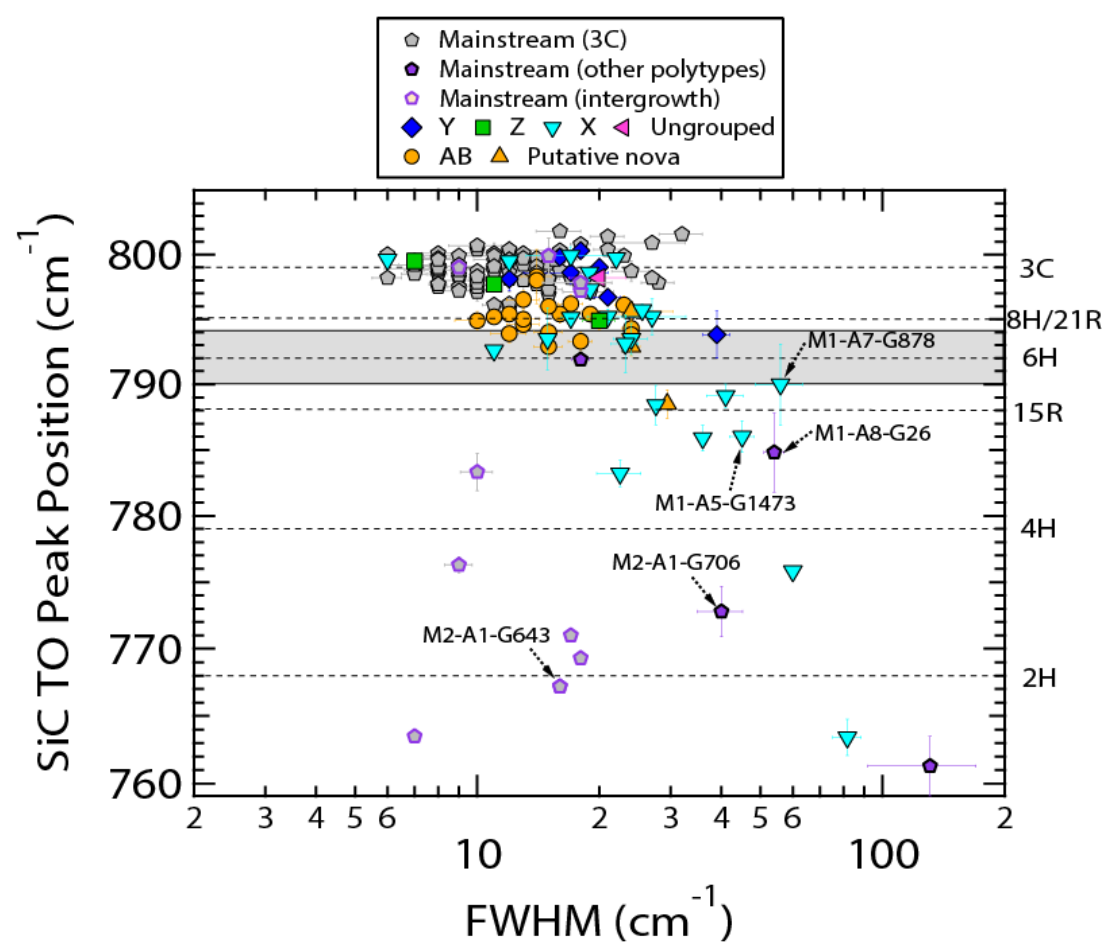

Figure 1. Raman peak position and width for 156 presolar SiC grains. The grains are classified by stellar progenitor type (mainstream AGB stars, type II supernovae (X, Y, Z), A-B, and possibly novae).

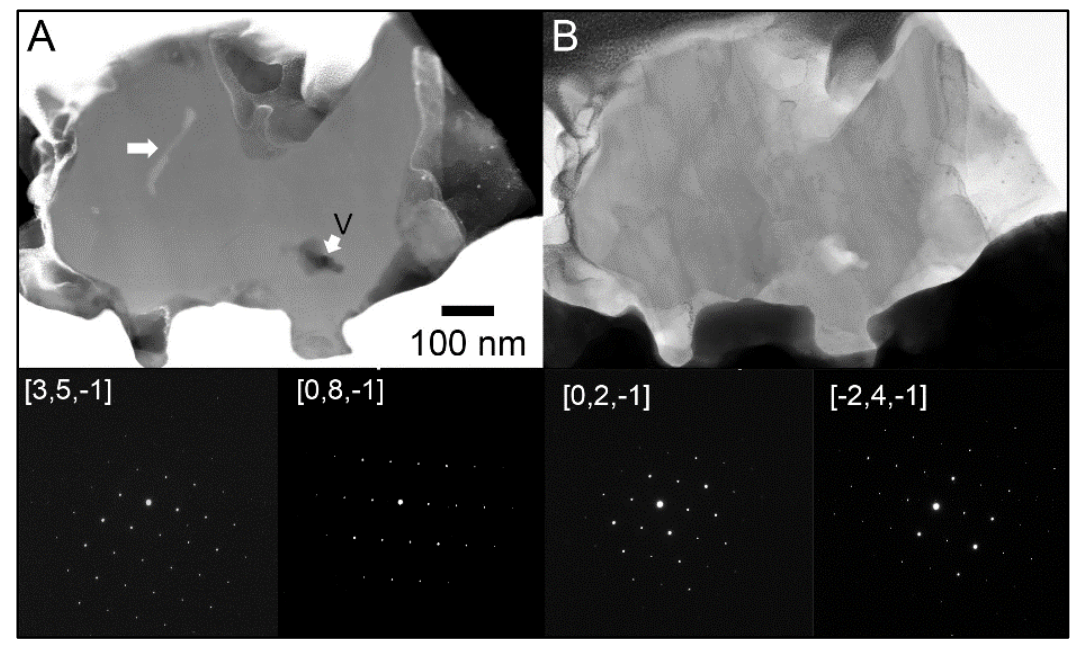

Figure 2. HAADF (A) and BF (B) STEM imaging and selected area diffraction of grain M1-A8-G26. Zones are indexed to the $8 \mathrm{H}$ polytype. The labes in (a) refer to a void (V) and a Ti, Al-rich subgrain (white arrow). 